\title{
Growth cone dynamics in the zebrafish embryonic forebrain is regulated by Brother of Cdo
}

\author{
Q1 James A. St John $n_{\wedge}^{\mathrm{a}, *}$, Susan Scott ${ }_{\wedge}^{\mathrm{a}}$, Kah Yau Chua $_{\wedge}^{\mathrm{b}}$, ${ }_{\wedge}$ Christina Claxton ${ }_{\wedge}^{\mathrm{b}}$, Brian Key $_{\wedge}^{\mathrm{b}}$ \\ a Eskitis Institute for Drug Discovery, Griffith University, Nathan 4111, Brisbane, Australia \\ ${ }^{\mathrm{b}}$ Brain Growth and Regeneration Laboratory, School of Biomedical Sciences, The University of Queensland, Brisbane 4072, Australia
}

\section{H I G H L I G H T S}

- Brother of Cdo is expressed around the supraoptic tract of zebrafish.

- Elavl3-eGFP labelling and time-lapse imaging revealed individual axon behaviour.

- Loss of Boc resulted in axons searching widely within the supraoptic tract.

- Boc acts to restrict axons within the confines of the supraoptic tract.

\section{A R T I C L E I N F O}

\section{Article history:}

Received 23 January 2013

Received in revised form 19 March 2013

Accepted 5 April 2013

\section{Keywords:}

Elavl3

Boc

Supraoptic tract

Time-lapse

Filopodia

\begin{abstract}
A B S T R A C T
During development of the embryonic zebrafish brain, the differential expression of axon guidance molecules directs the growth of axons along defined neuronal tracts. Neurons within the dorsorostral cluster of the presumptive telencephalon project axons ventrally along the supraoptic tract. Brother of Cdo (Boc) is a known axon guidance molecule that is expressed in a broad band lying ventral to the dorsorostral cluster of neurons. Loss of Boc function has previously been shown to perturb the development of the supraoptic tract. We have used live cell imaging of individual growth cones within the living zebrafish embryo to determine how Boc regulates the growth cone dynamics and axon guidance within the supraoptic tract. A plasmid construct encoding elavl3-eGFP was injected into early embryos to selectively label a small number of neurons while the expression of Boc was knocked down by injection of antisense morpholino oligonucleotides. Time-lapse imaging of growth cones within the living embryos revealed that loss of Boc significantly affected the morphology of growth cones in comparison to axons within control embryos. Growth cones navigating along the supraoptic tract in the absence of Boc extended significantly longer filopodia in the rostrocaudal direction. These results indicate that Boc acts to restrict axons and their filopodia within the narrow pathway of the supraoptic tract. The highly selective nature of these pathfinding defects reveal that Boc is likely to be one of many molecules that coordinate the trajectory of axons within the supraoptic tract.
\end{abstract}

(c) 2013 Elsevier Ireland Ltd. All rights reserved.

\section{Introduction}

During the development of dorsal telencephalon of the zebrafish, neurons localised within the dorsorostral cluster extend axons ventrally to form the supraoptic tract. The formation of this tract is likely to involve the restricted expression of numerous axon guidance molecules that act in concert to create domains which direct the trajectories of the axons. One of the molecules that has been implicated in axon guidance in this region is Brother of Cdo (Boc) [4] which is a receptor-like protein and a member of the

\footnotetext{
* Corresponding author. Tel.: +61 7 37353660; fax: +61 737354255. E-mail address: j.stjohn@griffith.edu.au (J.A. St John).
}

Ig superfamily with four Ig-like loops, three fibronectin type III repeats and a single transmembrane domain [8].

The binding and signalling partners of Boc are yet to be fully elucidated. Boc has been proposed to form a receptor complex with Cdo [8] but has also been shown to bind to sonic hedgehog (Shh). Retinal ganglion cell growth cones that express Boc are repelled from Shh in mouse forebrain [5]. Boc-Shh interactions have also been shown to directly regulate chemoattractive axon guidance during the formation of commissures in the spinal cord [10], the patterning of the ventral brain and spinal cord [1,2], and the formation of microcircuits within the cerebral cortex [6].

Within the developing zebrafish forebrain, boc is expressed in a highly restricted band that lies immediately ventral to the dorsorostral cluster of neurons [4]. Ventrally projecting axons from those neurons form the supraoptic tract which passes through the 
boc domain en route to the longitudinal axon tracts. It has been previously shown that loss of Boc from the developing brain causes a small number of axons that project along the supraoptic tract to inappropriately project caudally into virgin neuroepithlium [4]. Boc was proposed to act as a chemorepulsive molecule that restricted or confined the axons of the supraoptic tract to a narrow dorsoventral pathway. While it was clear that loss of Boc affected the growth of some axons, the majority of axons that entered the supraoptic tract successfully projected towards the longitudinal tracts in the ventral forebrain [4]. Thus, either the majority of axons either did not respond to Boc perturbation, or more subtle changes occurred that were not detected in fixed tissue preparations.

To determine how loss of Boc influences the behaviour of axons that remain within the supraoptic tract, we mosaically labelled small subpopulations of axons within the living embryo. We found that the loss of Boc resulted in axons within the supraoptic tract inappropriately exploring the local domain of the tract, but that despite these errors the axons were still able to correctly navigate the length of the supraoptic tract.

\section{Materials and methods}

\subsection{Fish maintenance}

Zebrafish (Danio rerio) were maintained on a 14-h light/10-h dark cycle. Embryos were naturally spawned through light induced mating and raised at $28.5^{\circ} \mathrm{C}$ in embryo medium [14]. Embryos were staged according to Kimmel et al. [9].

\subsection{Plasmid and morpholino construction and microinjection}

A $3.2 \mathrm{~kb}$ DNA fragment of $5^{\prime}$ UTR sequence of zebrafish Huc gene [11], containing the minimal promoter for neuron specific expression was amplified from genomic DNA using the following primers: elavl3 F: 5'-GAATTCACTAATTTGAAT-3' and elavl3 R: 5' TCTTGACGTACGTACAAAGATG-3'. The resulting PCR product was gel purified and TA cloned into the pGEM-T vector (Promega Corporation). The elavl3 promoter was excised from pGEM-T using flanking EcoRI sites and cloned directly into the EcoRI site of peGFP-1 vector (Clontech) to generate the elavl3:eGFP construct. Approximately $0.5-1 \mathrm{~nL}$ of solution containing 25-100 pg of DNA was injected directly into the cytoplasm of the cell.

Two 25-mer antisense morpholino (MO) oligonucleotides were designed to the $5^{\prime}$ UTR and signal sequence of zebrafish boc as described by Connor et al. [4]. As a control for specificity, a nonsense morpholino (Genetools LLC) was injected. The sequence for the control nonsense morpholino was $5^{\prime}$ CCTCTTACCTCAGTTACAATTTATA-3'. Concentrations of morpholino injected ranged from $0.25-10 \mathrm{mg} / \mathrm{ml}[0.5-1 \mathrm{nl}$ per embryo].

\subsection{Immunohistochemistry}

Embryos were fixed for $20 \mathrm{~min}$ in $4 \%$ paraformaldehyde and immunostained using IgG anti-acetylated $\alpha$-tubulin monoclonal antibody (1:200; ICN Biochemicals), as previously described [4]. Serial optical sections were collected with a Bio-Rad MRC-1024 laser scanning confocal microscope coupled to a Zeiss Axioplan microscope.

\subsection{Measurement of filopodia}

All measurements were obtained using SPOT software (Diagnostic Instruments, Sterling Heights, MI, USA) using calibrated calliper tools. Indicators of overall growth cone (GC) morphology included filopodial length and number of filopodia. The length of the filopodia was defined as the distance from the most distal visible portion of the filopodia to the exterior surface of the main ventral projecting axon or the growth cone. Pilot analyses of $n=9$ axons were performed blind and were consistent with comprehensive non-blind analyses on all samples.

\subsection{Time-lapse pricroscopy}

Embryos with grossly normal morphology at 18 hpf were anaesthetised in $0.01 \%$ Tricane (3-amino benzoic acid ethylester) (Sigma) and mounted on glass slides in $1 \%$ low melting temperature agarose. Images were captured using a dry $40 \times$ objective on an Olympus BX51 compound microscope using epifluorescence according to the procedure as described by St John and Key [12]. Images were taken at 10 second intervals for $1-5 \mathrm{~h}$ with $n=10-17$ sequences used for quantification. Measurements of the length of filopodia were made using SPOT software (Diagnostic Instruments, Sterling Heights, MI, USA). Data was analysed using ANOVA with a post hoc Student's $t$-test, with $p<0.05$ being considered as significant.

\section{Results}

\subsection{Mosaic labelling of neurons allows single axon resolution in} the supraoptic tract

Transient transgenic embryos were generated using a new transgene construct (elavl3-eGFP) in which the neuronal specific promoter elavl3 [11] drives expression of eGFP in neurons. Injection of $25 \mathrm{pg}$ of elavl3-eGFP did not label any neurons in the dorsorostral cluster whereas $37.5 \mathrm{pg}$ of elavl3-eGFP labelled dispersed neurons (arrows, Fig. 1B, $\mathrm{B}_{\wedge}^{\prime}$ ) which provided fine resolution of axons (arrowheads, Fig. 1B $\wedge$ ) with embryos showing normal gross morphologies rates $(>95 \%)$ similar to uninjected embryos. As the amount of elavl3-eGFP injected was increased to $50 \mathrm{pg}$ more neurons were labelled in the dorsorostral cluster (arrows, Fig. 1C, $\mathrm{C}$ ) $)$. Axons were also detected in other tracts (see labelling in the post-optic commissure, Fig. 1B, $\mathrm{B}^{\prime}$ ). When injected with $100 \mathrm{pg}$ of Huc-eGFP, $19 \%$ $(n=52)$ of embryos exhibited morphological defects (body torsion, tail curling and abnormal head shape). We subsequently used $37.5 \mathrm{pg}$ of elavl3-eGFP for the following experiments as this best provided the single cell labelling within the dorsal-rostral cluster that we desired and we used only those embryos exhibiting normal gross morphology.

\subsection{Growth cone shape changes throughout the supraoptic tract}

In wild-type animals, axons entered the supraoptic tract directly by projecting ventrally and without evidence of exploratory growth towards the dorsal surface of the brain (Fig. 1D, $\mathrm{D} /$ ). The growth cones exhibited complex shapes as they grew towards the supraoptic tract (arrowheads, Fig. 1E, E⿰冫). Upon entering the supraoptic tract the growth cones became streamlined (arrowheads, Fig. 1F, $\mathrm{F} \wedge$ ). Axons retained complex protrusions close to the cell body near the entry into the supraoptic tract (arrows with tail, Fig. $1 \mathrm{~F}_{\curlywedge}^{\prime}, \mathrm{G}^{\prime}$ ) while the part of the axon within the supraoptic tract remained unbranched and linear in trajectory (Fig. $1 \mathrm{~F}^{\prime}, \mathrm{G}^{\prime}$ ). Upon reaching the junction with the tract of the post-optic commissure the growth cones enlarged (arrowheads, Fig. 1G, Gi; compare with arrowheads in Fig. 1 $\left.\mathrm{F}^{\prime}\right)$.

\subsection{Live cell imaging of individual axons in supraoptic tract}

It has been previously shown that boc is expressed in the region ventral to the dorsorostral cluster and that supraoptic tract axons 

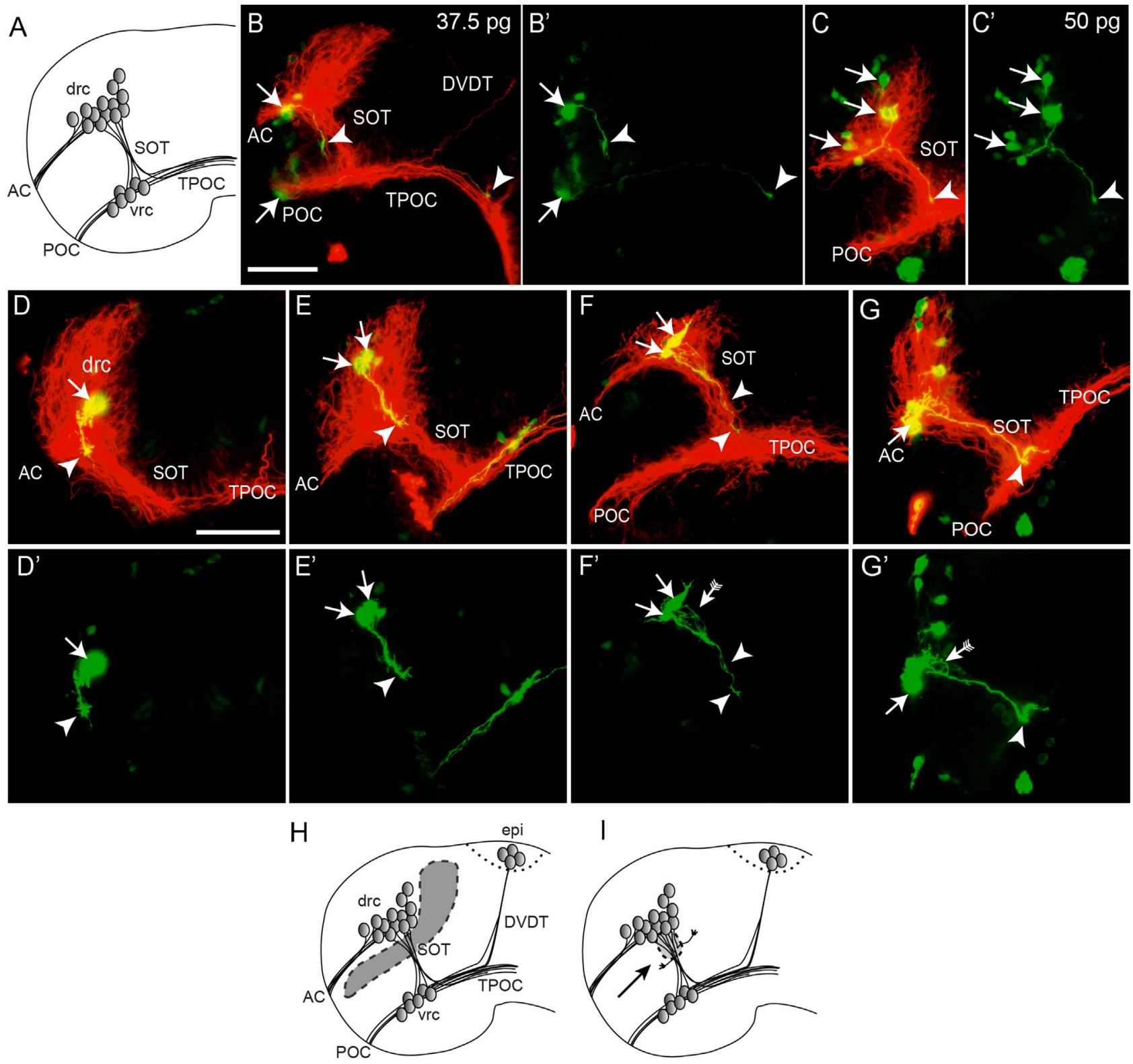

BOC Expression

\section{Area affected by \\ $\because$ loss of function}

Fig. 1. Mosaic expression of eGFP in embryonic zebrafish brain (20-25 hpf). Lateral views of the zebrafish brain show eGFP (green) and acetylated $\alpha$-tubulin immunostaining (red); or eGFP alone in adjacent images. Anterior is to the left and dorsal to the top as indicated in panel A. Single-cell embryos were injected with (B, B $\left.{ }^{\prime}\right) 37.5 \mathrm{pg}$ elavl3-eGFP; (C, C) 50 pg elavl3-eGFP. (D-G) Neurons (arrows) within the dorsal-rostral cluster (drc) projected axons ventrally into the supraoptic tract (SOT); growth cones (arrowheads) were initially complex $\left(D, D_{\mu}^{\prime}, E, E^{\prime}\right)$, became bullet-shaped $\left(F, F^{\prime}\right)$, and then more complex after exiting the supraoptic tract $\left(G^{\prime}, G^{\prime}\right)$. Scale bar in $B$ is $50 \mu m$ and refers to $(B)$ and (C); scale bar in (D) is $50 \mu \mathrm{m}$ and refers to (D)- $(\mathrm{G})$. (H) BOC is expressed in a broad band (dashed region) underlying the dorsal-rostral cluster; axons from the dorsal-rostral cluster extend through the BOC region to form the supraoptic tract. (I) In embryos injected with antisense morpholinos against BOC, some axons within the dorsal region of the supraoptic tract aberrantly project caudally out of the supraoptic tract. Time-lapse imaging focused on the region indicated by the arrow. AC, anterior commissure; POC, post-

Q3 optic commissure; TPOC, tract of the post-optic commissure; DVDT, dorsoventral diencephalic tract, vrc, ventrorostral cluster; epi, epiphyseal neurons. (For interpretation of the references to colour in this figure legend, the reader is referred to the web version of this article.)

project through the Boc domain (Fig. 1H) [4]. In embryos in which Boc expression was knocked down, some axons failed to properly navigate along the supraoptic tract and exited the dorsal region of the supraoptic tract and aberrantly projected posteriorly (Fig. 1I) [4]. We have now used the elavl3-eGFP construct to visualise axon behaviour in living embryos to determine how the loss of Boc affects axon navigation at the individual growth cone level. Our analysis was centred on the dynamics of the growth cone within the supraoptic tract (arrow in Fig. 1I) overlying the Boc expression domain, as this was where aberrant axon projections were previously detected.

We first determined the behaviour of growth cones within the supraoptic tract of wild-type embryos. Wild-type growth cones progressed with very few pauses during migration (Fig. 2A). Growth cones were elongated and small filopodia extended in both anterior and caudal directions (arrowheads, Fig. 2A). Interestingly, filopodia were observed up to $10 \mu \mathrm{m}$ in length (see below for quantification) indicating that growth cones in the supraoptic 

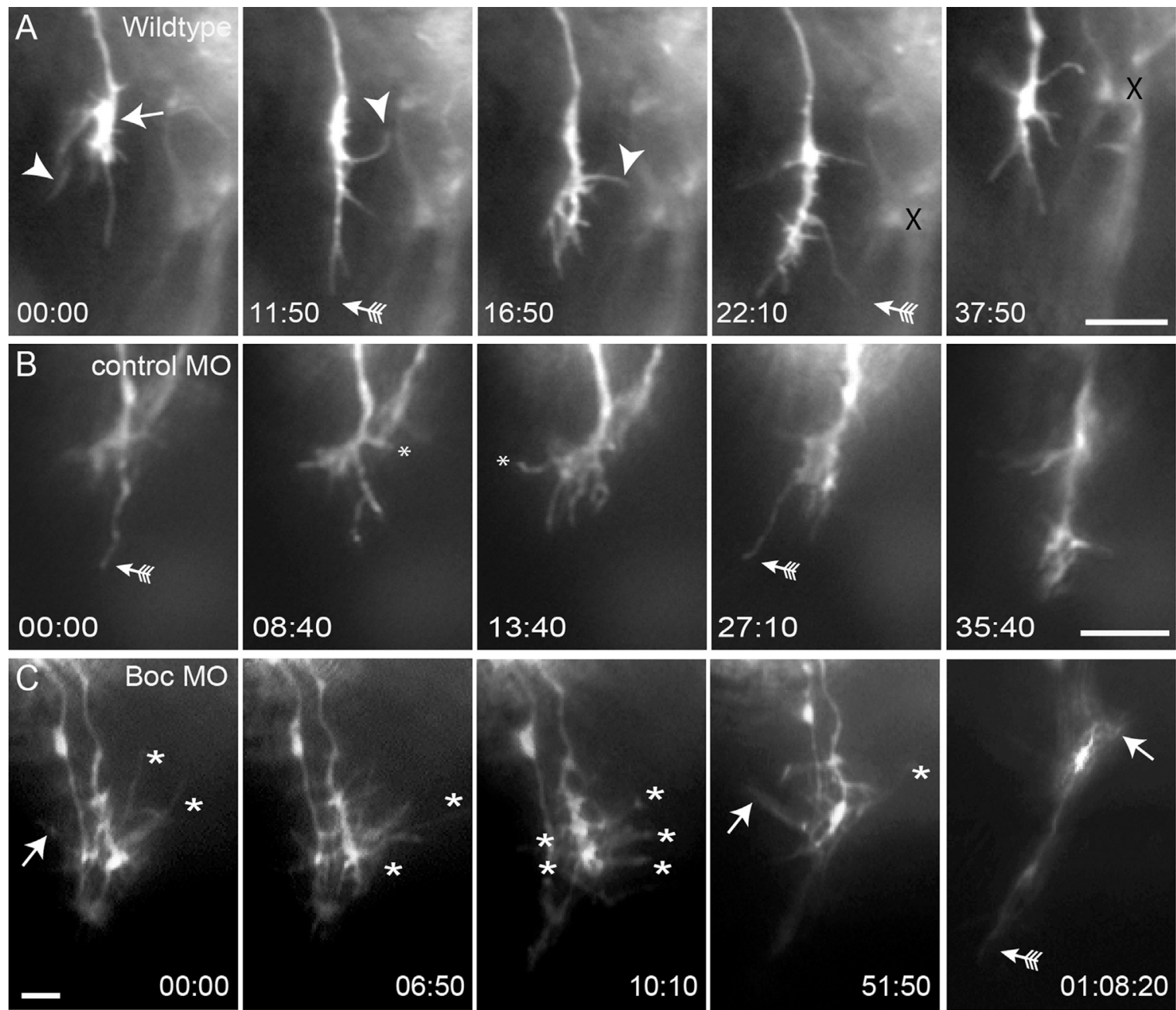

$35: 40$
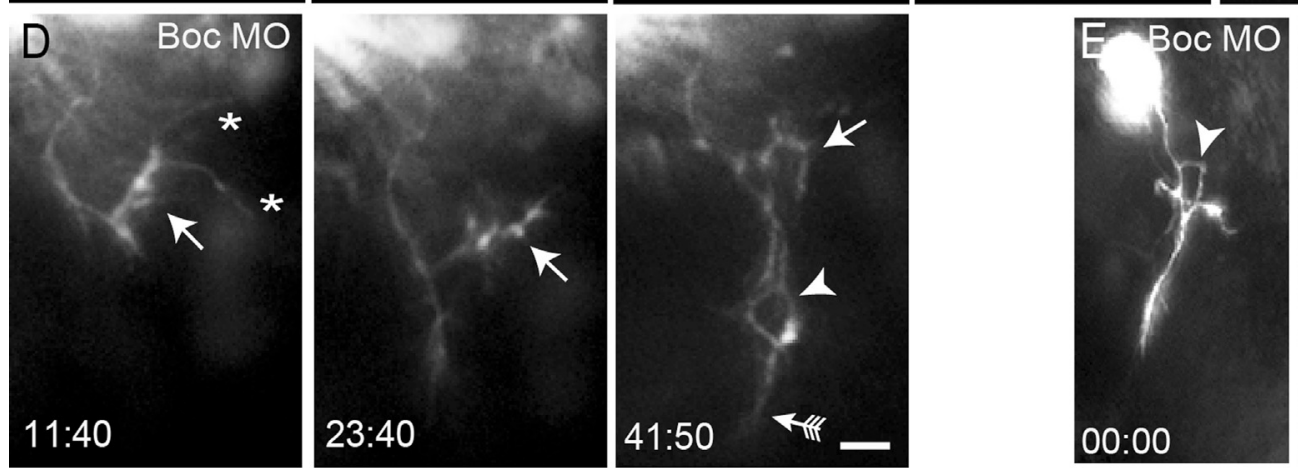

Yili

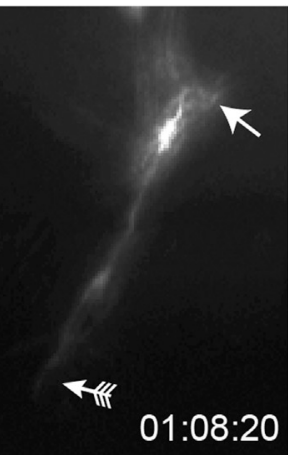

F distribution of filopodia

$\mathrm{G}$ longest filopodia
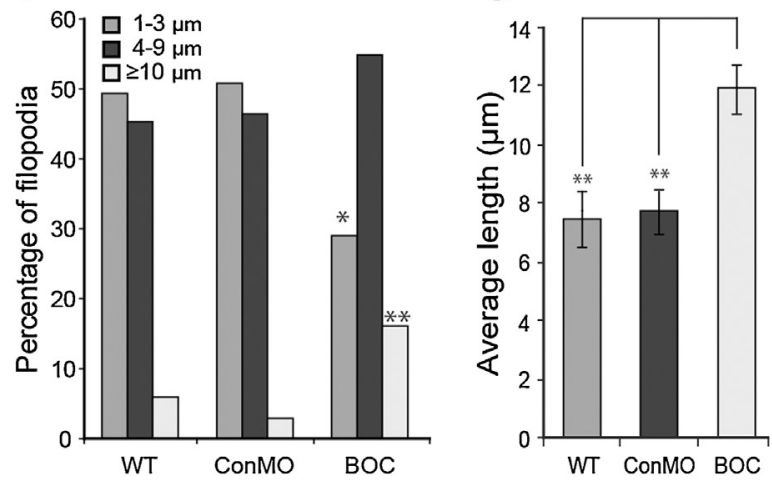

Please cite this article in press as: J.A. St John, et al., Growth cone dynamics in the zebrafish embryonic forebrain is regulated by Brother of Cdo, Neurosci. Lett. (2013), http://dx.doi.org/10.1016/j.neulet.2013.04.009 
tract were capable of sampling almost the complete width of the supraoptic tract, which was approximately $20 \mu \mathrm{m}$ in diameter (as determined from immunostained preparations in Fig. 1). The majority of the longest filopodia extended in the ventral direction (arrows with tail, Fig. 2A).

Next we examined embryos that had been injected with control morpholinos. The growth cones exhibited behaviour similar to that of axons in wild-type embryos with the longest filopodia projecting in the ventral direction (arrows with tail, Fig. 2B; Movies 1 and 2). While some filopodia projected in the anterior and posterior directions (asterisks, Fig. 2B) they were shorter than the ventrally projecting filopodia. Filopodia were actively extending and retracting around the whole circumference of both old and new growth cones as the axon elongated.

We next examined embryos that had been injected with two non-overlapping boc antisense morpholinos as previously described [4], both of which produced similar phenotypes. In these animals, the behaviour of the growth cones was distinctly different to that observed in control embryos (Movies 3 and 4). The axons typically bifurcated (arrowheads, Fig. 2D and E), had multiple branches that projected either rostrally or caudally (arrows, Fig. 2C-E) and prominent filopodia that extended both rostrally and caudally from these branches (asterisks, Fig. $2 \mathrm{C}$ and $\mathrm{D}$ ) in addition to those that normally extended ventrally (arrows with tail, Fig. 2C-E). When viewed in time-lapse, growth cones with multiple filopodia that selectively extended and retracted along the anterior-posterior axis appeared to remain stationary (Movie 3). Movie 4 is an example of a supraoptic tract axon that has bifurcated and possesses at least two active growth cones, one of which extended caudally consistent with the aberrant extension of axons that we have previously described [4]. Despite the abnormal branching the axons continued to project ventrally (arrow with tail, Fig. 2E) although there did appear to be excessive filopodial activity on the axon shaft.

We quantified some characteristics of the growth cones in embryos that provided clear visualisation of the filopodia. We measured the length of filopodia and placed them in three categories $1-3 \mu \mathrm{m}, 4-9 \mu \mathrm{m}$ and $\geq 10 \mu \mathrm{m}$. Embryos injected with boc morpholinos had significantly fewer $(p<0.05)$ filopodia in the shortest category and significantly greater $(p<0.01)$ proportion of filopodia in the longest category compared to wild-type and morpholino control embryos (Fig. 2F; $n=17$ WT; $n=10$ Control MO; $n=17$ Boc MO). For filopodia that extended more than $10 \mu \mathrm{m}$ there was no directional preference of their trajectory with aberrant filopodia being present on both the rostral and caudal regions of the supraoptic tract (WT $=55 \%$ in caudal direction, Control $\mathrm{MO}=46.7 \%$ in caudal direction, $\mathrm{Boc} \mathrm{MO}=52.9 \%$ in caudal direction; $n=0-32$ filopodia in each of 5 embryos in all groups). The length of the longest filopodia on axons in boc morpholino embryos was significantly greater $(p<0.01)$ than the longest filopodia on axons in the wild-type and control embryos (Fig. 2G; $n=17 \mathrm{WT} ; n=10$ Control MO; $n=17$ Boc MO).

\section{Discussion}

Our results have revealed the dynamics of growth cone extension within the supraoptic tract. Loss-of-function of Boc from neuroepithelial cells underlying the entry point of telencephalic axons into the supraoptic tract disrupted the morphology of growth cones. Growth cones bifurcated and aberrant branches were established which led to inappropriate growth of axons into diencephalic regions normally devoid of axons.

Knock down of Boc causes a selective defect in the guidance of a subpopulation of axons coursing in the supraoptic tract that forms the first connection between the telencephalon and diencephalon [4]. Rather than growing directly from the dorsal telencephalon towards the ventral diencephalon, some supraoptic tract axons are diverted caudally away from the narrow confines of their ventrodorsal trajectory with knock down of Boc (Fig. $2 \mathrm{~N}_{-} \mathrm{O}$ ). These aberrant axons wander through a neuroepithelial domain that normally expresses boc. These results led to the idea that the Boc-expressing neuroepithelial domain, which begins at the point where axons of the dorsorostral cluster of neurons enter the supraoptic tract, was inhibitory to these supraoptic tract axons and acted like a surround barrier to channel axons ventrally towards the major longitudinal axon tract coursing along the rostrocaudal axis of the brain.

Since the complexity of growth cone morphology and the dynamics of axon extension reflect the nature of the guidance cues in the microenvironment we first decided to examine the shape of supraoptic tract growth cones throughout their trajectory. By injecting large numbers of embryos with the Huc-eGFP plasmid it was possible to obtain specimens with discrete labelling of dorsorostral cluster neurons with axons projecting into and along the supraoptic tract.

Using fixed tissue analyses, we have previously shown that knock down of BOC caused some axons to inappropriately extend out of the supraoptic tract in the caudal direction [4]. We therefore reasoned that this phenotype may actually represent one end of a spectrum of abnormal growth behaviours by supraoptic tract axons. To test this hypothesis we examined axon growth at the single cell resolution in vivo by time-lapse microscopy of living embryos. We found that knock down of Boc caused axon splitting and branching as depicted in the fixed preparations and that there were more dynamic filopodia. In addition, the length of the filopodia of the ventrally directed growth cones were longer in the Boc loss-of-function embryos compared to controls. These subtle defects could not be identified in fixed preparations and were not visible in whole-mounted embryos labelled for expression of acetylated $\alpha$-tubulin which stains all axons [4]. Thus, Boc influences a range of growth behaviours in the supraoptic tract including axon guidance, axon splitting and filopodial extensions.

It is not immediately clear how boc regulates axon guidance and growth cone morphology in the supraoptic tract. Cell transplant experiments are sometimes performed in zebrafish to create chimaeras in order to elucidate the role of molecules of interest. However, in our study we considered that it would not be appropriate to transplant the supraoptic tract neurons since the supraoptic tract neurons themselves do not express Boc, but instead their axons migrate through Boc domains [4]. Thus we could not use this approach to determine more about the potential signalling pathways. We propose that Boc must be acting indirectly to modulate supraoptic tract axon guidance. The evidence points towards Boc regulating the chemorepulsiveness of the neuroepithelium.

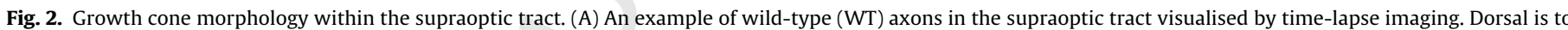

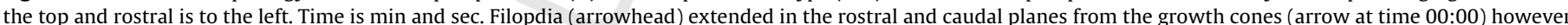

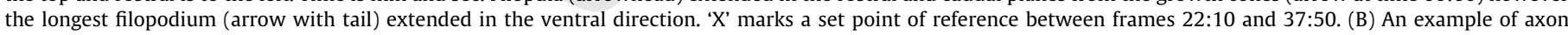

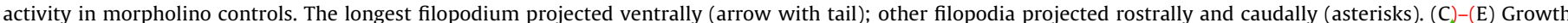

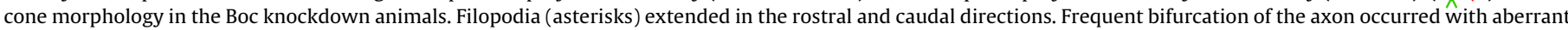

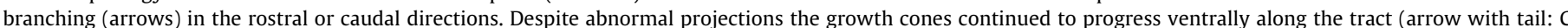

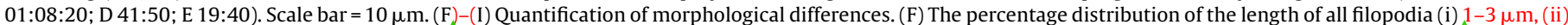

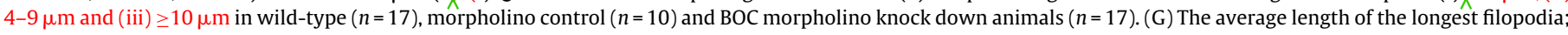
wild-type $n=17$, morpholino control $n=10$ and BOC morpholino knock down animals $n=17$. ${ }^{*} p<0.05,{ }^{* *} p<0.01$. 
In the absence of Boc this neuroepithelium appears to be conducive to axon growth cone and to even modulate growth cone behaviour remotely within the supraoptic tract. One possibility is that Boc acts a sink for ventrally expressed Shh and concentrates it dorsally where it acts as a chemorepulsive ligand for supraoptic tract axons. Cdo, another Shh receptor, is expressed dorsally in the telencephalon and may be directly responsible for this behaviour in supraoptic tract axons $[1,10,13,15]$. The possibility that Shh is chemorepulsive to supraoptic tract axons is consistent with our previous observations that supraoptic tract axons never enter the ventral domain of neuroepithelial cells expressing Shh [7]. Another possibility is that boc, which is expressed weakly throughout the early forebrain [4], is affecting initial patterning of the ventral forebrain which then indirectly affects axon guidance in the later developing supraoptic tract. This seems unlikely since neither the gross patterning of the forebrain [2] nor the overall topography of the scaffold of axon tracts [4] appears perturbed following antisense knock down of boc. In contrast, the point mutation in the zebrafish umleitung mutant, that generates a truncated version of Boc, seems to be more severely penetrant and indirectly affects axon guidance in the retinotectal pathway because of early ventral patterning defects [2]. The molecular mechanisms underlying the role of boc, cdo and shh in the development of supraoptic tract now needs to be defined by molecular genetic interaction studies in zebrafish.

Interestingly, in mice Boc is expressed on the axons of spinal commissural neurons and acts as a chemoattractive receptor for Shh [10]. However, Boc was subsequently shown to mediate the chemorepulsive response of retinal axons to Shh [5]. Despite totally different cellular responses, in both cases Boc was expressed by axons and shown to directly modulate axon guidance in response to Shh. As discussed above, we consider that other factors or coreceptors are likely to be involved, however future work could examine the involvement of the hedgehog pathway in supraoptic tract-Boc axon guidance by examining the guidance of the supraoptic tract axons in hedgehog mutants or by blocking the hedgehog pathway with selective inhibitors such as cyclopamine [3].

\section{Conclusion}

We found that in the absence of Boc, axons within the supraoptic tract exhibited aberrant growth behaviours within the local domain of the tract, but these were insufficient to cause gross mistargeting and the axons were able to correctly navigate ventrally. These results reveal that the failure to observe axon guidance phenotypes in some loss-of-gene-function experiments may be explained both by the transient nature of the cellular response, and by the fact that growth cone defects are not resolvable within the milieu of the whole axon tract. In such cases, real-time analysis and single cell resolution are needed for detection of phenotypes.

\section{Acknowledgements}

This work was supported by grants from the Australian N.H.M.R.C. to JStJ and BK and from the Australian Research Council to B.K.

\section{Appendix A. Supplementary data}

Supplementary data associated with this article can be found, in the online version, at http://dx.doi.org/10.1016/ j.neulet.2013.04.009.

\section{References}

[1] B.L. Allen, J.Y. Song, L. Izzi, I.W. Althaus, J.S. Kang, F. Charron, R.S. Krauss A.P. McMahon, Overlapping roles and collective requirement for the coreceptors GAS1, CDO, and BOC in SHH pathway function, Dev, Cell 20 (2011) 775-787.

[2] S.A. Bergeron, O.V. Tyurina, E. Miller, A. Bagas, R.O. Karlstrom, Brother of cdo (umleitung) is cell-autonomously required for Hedgehog-mediated ventral CNS patterning in the zebrafish, Development 138 (2011) 75-85.

[3] C. Brusegan, A. Pistocchi, A. Frassine, I. Della Noce, F. Schepis, F. Cotelli, Ccdc80-11 Is involved in axon pathfinding of zebrafish motoneurons, PLoS ONE 7 (2012) e31851.

[4] R.M. Connor, C.L. Allen, C.A. Devine, C. Claxton, B. Key, BOC, brother of CDO, is a dorsoventral axon-guidance molecule in the embryonic vertebrate brain, $\mathrm{J}_{\Lambda}$ Comp. Neurol. 485 (2005) 32-42.

[5] P.J. Fabre, T. Shimogori, F. Charron, Segregation of ipsilateral retinal ganglion cell axons at the optic chiasm requires the Shh receptor Boc, J Neurosci. 30 (2010) 266-275.

[6] C.C. Harwell, P.R. Parker, S.M. Gee, A. Okada, S.K. McConnell, A.C. Kreitzer, A.R. Kriegstein, Sonic hedgehog expression in corticofugal projection neurons directs cortical microcircuit formation, Neuron 73 (2012) 1116-1126.

[7] J.T. Hjorth, B. Key, Are pioneer axons guided by regulatory gene expression domains in the zebrafish forebrain? High-resolution analysis of the patterning of the zebrafish brain during axon tract formation, Dev Biol. 229 (2001) 271-286.

[8] J.S. Kang, P.J. Mulieri, Y. Hu, L. Taliana, R.S. Krauss, BOC, an Ig superfamily member, associates with CDO to positively regulate myogenic differentiation, EMBO J, 21 (2002) 114-124.

[9] C.B. Kimmel, W.W. Ballard, S.R. Kimmel, B. Ullmann, T.F. Schilling, Stages of embryonic development of the zebrafish, Dev, Dyn. 203 (1995) 253-310.

[10] A. Okada, F. Charron, S. Morin, D.S. Shin, K. Wong, P.J. Fabre, M. Tessier-Lavigne S.K. McConnell, Boc is a receptor for sonic hedgehog in the guidance of commissural axons, Nature 444 (2006) 369-373.

[11] H.C. Park, C.H. Kim, Y.K. Bae, S.Y. Yeo, S.H. Kim, S.K. Hong, J. Shin, K.W. Yoo, M Hibi, T. Hirano, N. Miki, A.B. Chitnis, T.L. Huh, Analysis of upstream elements in the HuC promoter leads to the establishment of transgenic zebrafish with fluorescent neurons, Dev, Biol. 227 (2000) 279-293.

[12] J.A. St John, B. Key, HûC-eGFP mosaic labelling of neurons in zebrafish enables in vivo live cell imaging of growth cones, J Mol. Histol. 43 (2012) 615-623.

[13] T. Tenzen, B.L. Allen, F. Cole, J.S. Kang, R.S. Krauss, A.P. McMahon, The cell surface membrane proteins Cdo and Boc are components and targets of the Hedgehog signaling pathway and feedback network in mice, Dev, Cell 10 (2006) 647-656.

[14] M. Westerfield, The Zebrafish Book: A Guide for the Laboratory Use of Zebrafish (Brachydanio rerio), University of Oregon Press, Eugene, OR, 1994.

[15] W. Zhang, J.S. Kang, F. Cole, M.J. Yi, R.S. Krauss, Cdo functions at multiple points in the Sonic Hedgehog pathway, and Cdo-deficient mice accurately model human holoprosencephaly, Dev Cell 10 (2006) 657-665. 